\title{
A comparison among Polynomial model, Reduced polynomial model and Ogden model for polyurethane foam
}

\author{
Minglei Ju ${ }^{1, a}$, Hamdi Jmal ${ }^{1, b}$, Raphaël Dupuis ${ }^{1, c}$, Evelyne Aubry ${ }^{1, d}$ \\ ${ }^{1}$ Laboratoire MIPS, Université de Haute Alsace, 68093 Mulhouse, FRANCE. \\ a minglei.ju@uha.fr, ${ }^{b}$ hamdi.jmal@uha.fr, ${ }^{c}$ Raphael.dupuis@uha.fr, ${ }^{d}$ evelyne.aubry@uha.fr
}

Keywords: Polyurethane foam; Quasi-static behaviour; Elasticity; Viscoelasticity; Identification.

\begin{abstract}
Polyurethane foam is widely used in numerous applications. The quasi-static mechanical behaviour of polyurethane foam shows a nonlinear elasticity and a viscoelasticity in large compression deformation. In this paper, the uniaxial compression / decompression tests in three different strain rates are performed. The purpose of this paper is to propose three visco-hyperelastic models and to identify the parameters based on the experimental results. A difference-stress proper identification method is used to obtain the model parameters. The results show that the three models can predict the mechanical behaviour of polyurethane foam very well. Finally, the three models are compared according to the model results.
\end{abstract}

\section{Introduction}

The variety of applications of polyurethane foams in different industrial areas is enormous. The mechanical behaviour of polyurethane foams under different strain rates and large strain level is complicated. The stress-strain response of polyurethane foams under uniaxial quasi-static compression shows nonlinear hyperelastic behaviour and viscoelastic behaviour. In the literature, many models have been proposed to describe hyperelastic behaviour for materials. Mooney proposed a model with two parameters [1]. Rivlin made a modification of the Mooney model and proposed a general expression which named Mooney-Rivlin model [2, 3]. In 1972, Ogden [4, 5] proposed a strain energy function expressed in terms of principal stretches, which is a very general expression for describing hyperelastic materials. But all these models cannot describe the difference stress behaviour between loading and unloading. Polyurethane foams also show a significant hysteretic response during unloading after prior loading in uniaxial compression. The stress on unloading is significantly lower than that during loading at the same strain. Because of the viscoelastic behaviour, the polyurethane foam cannot recover immediately after the loading is released and the current status is affected by the previous loading history. In order to indicate this phenomena, Yang et al. [6] developed a visco-hyperelastic model to capture the viscoelastic behaviour of foams. This model is adopted for high strain rate but it cannot predict the behaviour of unloading phase and it also cannot be used in the study of quasi-static mechanical behaviour.

This paper presents three hyperelastic constitutive models for the description of the hyperelastic part of polyurethane foams. A memory model is proposed here to study the viscoelastic behaviour of the polyurethane foams. A difference-stress identification method has been carried out to obtain the dimensionless viscoelastic parameters. The corresponding identification errors are taken into account to analyze the results.

\section{Experiments}

Polyurethane foam, which has similar characteristics to those of automotive seat foam, referred to here as foam A, was considered in the present work. The test specimens were cut from a foam block $2000 \mathrm{~mm} \times 1200 \mathrm{~mm} \times 75 \mathrm{~mm}$ (length, breadth, thickness). All specimens were provided with the same mechanical and environmental histories. A series of uniaxial compression/decompression tests were carried out at a constant temperature and humidity with a standard compression-tension testing device instron 3300r4204 driven with bluehill software. The test machine includes a basis frame and an upper block which moves vertically. Two compression plates were installed: one on the base 
of the machine and the other on the force sensor of the crosshead. The two plates were carefully checked to be strictly parallel. For the sake of minimization of the noise contribution, the maximum experimental response force of foam had to be slightly less than the load cell maximum capacity. The test conditions (Table 1) were conducted using the bluehill software configuration window. The test started when the upper plate began to affect the foams and then the upper block moved down to compress the samples to the final level. The final compression ratio was $80 \%$ of the original thickness. The minimum and maximum experimental envelopes can be extracted by $\mathrm{N}$ tests.

Table 1. Quasi-static compression test conditions

\begin{tabular}{|l|l|l|l|l|l|l|l|}
\hline & $\mathrm{N}_{\text {cyc }}$ & $\dot{\varepsilon}\left[s^{-1}\right]$ & $\varepsilon_{0}[\%]$ & $\varepsilon_{\max }[\%]$ & $\mathrm{T}[\mathrm{sec}]$ & $\mathrm{T}_{\text {ech }}[\mathrm{sec}]$ & $\mathrm{N}$ \\
\hline Test 1 & 1 & $1.0610^{-2}$ & 0 & 80 & 150 & 0.0625 & 17 \\
\hline Test 2 & 1 & $5.3310^{-3}$ & 0 & 80 & 300 & 0.125 & 37 \\
\hline Test 3 & 1 & $6.6610^{-4}$ & 0 & 80 & 2400 & 2 & 15 \\
\hline
\end{tabular}

Fig. 1 shows the experimental stress-strain result of polyurethane foam with three different strain rates. It can be seen in Fig. 1 that the deformation of polyurethane foam has three regions, which can be distinguished during uniaxial compression: initial elastic deformation, collapse deformation and compaction deformation. Fig. 1 (b) also indicates that the strain rates affect the experimental curves and the experimental stress-strain for loading and unloading phase is quite different due to the viscoelastic properties.
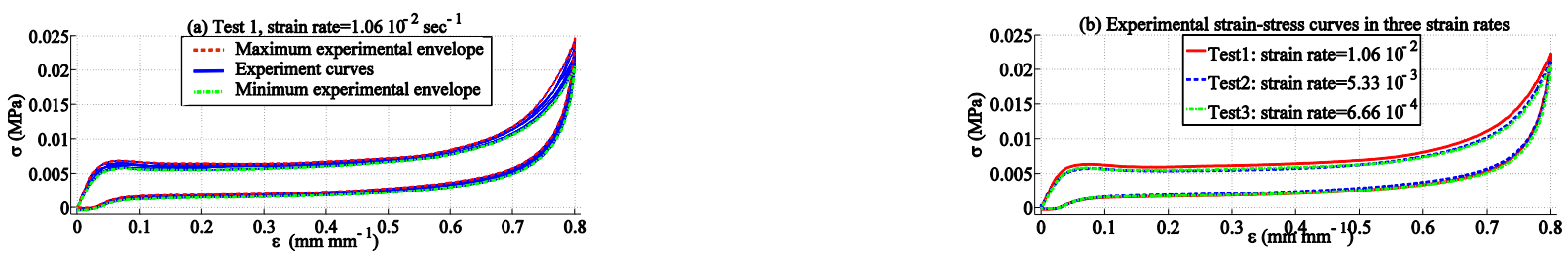

Fig.1 Experimental results. a. test 1; b. Influence of strain rates

\section{Modelling}

According to the experimental results mentioned in previous section and the empirical results, polyurethane foams exhibit nonlinear hyperelastic and viscoelastic properties. The strain energy function, denoted $W=W(F)$ or $W=W\left(\lambda_{1}, \lambda_{2}, \lambda_{3}\right)$, where $\mathrm{F}$ is the deformation tensor, $\lambda_{i}(i=1,2,3)$ are the principal stretches and given by $\lambda_{i}=1+\varepsilon_{i}$, where $\varepsilon_{i}$ are strains (for compression $\varepsilon_{i}<0$ ), is commonly used to describe the hyperelastic phenomenon. In the present paper, the strain energy functions with principal stretches are used and the stress can be derived from the function by:

$$
\sigma_{i}=\frac{\lambda_{i}}{J} \frac{\partial W}{\partial \lambda_{i}}
$$

The hyperelastic constitutive model developed by Ogden [5] is widely used for compressible materials and the stored energy, $\mathrm{W}$, is expressed in the form of a series:

$$
W=\sum_{n=1}^{N} \frac{\mu_{n}}{\alpha_{n}}\left(\lambda_{1}^{\alpha_{n}}+\lambda_{2}^{\alpha_{n}}+\lambda_{3}^{\alpha_{n}}-3\right)+F(J)
$$

where $\mu_{n}, \alpha_{n}$ are material parameters, $F(J)$ is the volumetric function. Based on Eq. 1 and Eq. 2, the stress for Ogden model can be obtained:

$$
\sigma_{2}=\lambda_{2}^{-1} \sum_{n=1}^{N} \mu_{n}\left(\lambda_{2}^{\alpha_{n}}-1\right)
$$

The polynomial hyperelastic material model is a phenomenological model of rubber elasticity. This model also can describe the elastic properties of compressible materials. The strain energy density function for the polynomial model is:

$$
W=\sum_{i, j=1}^{N} C_{i j}\left(\bar{I}_{1}-3\right)^{i}\left(\bar{I}_{2}-3\right)^{j}+\sum_{i=1}^{N} D_{i}(J-1)^{2 i}
$$

where 


$$
\overline{I_{1}}=J^{-2 / 3} I_{1} ; I_{1}=\lambda_{1}^{2}+\lambda_{2}^{2}+\lambda_{3}^{2} ; \bar{I}_{2}=J^{-4 / 3} I_{2} ; I_{2}=\lambda_{1}^{2} \lambda_{2}^{2}+\lambda_{2}^{2} \lambda_{3}^{2}+\lambda_{1}^{2} \lambda_{3}^{2} ; J=\operatorname{det}(F)
$$

When $\mathrm{N}=1$, the model reduces to the compressible Mooney-Rivlin model.

Particular forms of the Eq. 4 can be obtained by setting specific coefficients to zero. If all $C_{i j}$ with $j \neq 0$ are set to zero, the reduced polynomial form is obtained:

$$
W=\sum_{i=1}^{N} C_{i 0}\left(\overline{I_{1}}-3\right)^{i}+\sum_{i=1}^{N} D_{i}(J-1)^{2 i}
$$

By setting $\mathrm{N}=1$, the Neo-Hookean form is recovered.

In the literature, a variety of macroscopic models are used to describe the viscoelastic behaviour, such as the Maxwell model, the Kelvin Voigt model and the Zener model [7], etc. In this paper, the viscoelastic stress is represented by a hereditary integral model:

$$
\sigma_{\text {viscoelastic }}=\int_{0}^{t} \sum_{i=1}^{I} a_{i} e^{-\beta_{i}(t-\tau)} \varepsilon(\tau) d \tau
$$

This model takes account of the memory criterion of the polyurethane foams and can indicate the viscoelastic properties during the compression test process. Combining the elastic component and the viscoelastic component, the global visco-hyperelastic model of polyurethane foams undergoing large compressive deformation can be given by:

$$
\sigma=\sigma_{\text {elastic }}+\sigma_{\text {viscoelastic }}
$$

For the quasi-static compression test, the strain rates are a constant in the loading and unloading process. So, the relation between strain and the time is linear. Based on the symmetry of the elastic stress during the loading and unloading phases, the difference-stress method is used to identify the viscoelastic parameters [7]. The value of I in Eq. 7 is set to 2 depending on the comparison result of different value of I. So, the difference stress between loading and unloading phases can be obtained:

$$
\begin{aligned}
\delta \sigma_{\text {viscoelastic }} & =\sigma_{v_{-} L}-\sigma_{v_{-} U L} \\
& =\int_{0}^{t_{L}} \sum_{i=1}^{2} a_{i}^{L} e^{-\beta_{i}^{L}\left(t_{L}-\tau\right)} \varepsilon_{L}(\tau) d \tau-\int_{0}^{\frac{T}{2}} \sum_{i=1}^{2} a_{i}^{L} e^{-\beta_{i}^{L}\left(t_{U L}-\tau\right)} \varepsilon_{L}(\tau) d \tau-\int_{\frac{T}{2}}^{t_{U L}} \sum_{i=1}^{2} a_{i}^{U L} e^{-\beta_{i}^{U L}\left(t_{U L}-\tau\right)} \varepsilon_{U L}(\tau) d \tau
\end{aligned}
$$

where $t_{L} \in[0, \mathrm{~T} / 2]$ and $t_{U L} \in[\mathrm{T} / 2, \mathrm{~T}]$ are the loading time and unloading time, respectively.

Eq. (9) was considered to identify the viscoelastic parameters. After getting hold of the viscoelastic stress in the loading and unloading phases, the elastic stress is determinate by using the hyperelastic models mentioned above (Ogden, polynomial and reduced polynomial).

The optimization methods are used as basic tools to identify both viscoelastic parameters and elastic parameters. The stress-strain experimental data can be obtained from the uniaxial compression tests. The mean square error between the experiment data and model data can be calculated using a function $f$ :

$$
f=\frac{1}{n} \sqrt{\sum_{i=1}^{n}\left[\sigma_{m}\left(\varepsilon_{e}\right)-\sigma_{e}\left(\varepsilon_{e}\right)\right]^{2}}
$$

where $\sigma_{m}\left(\varepsilon_{e}\right)$ are the model stress and $\sigma_{e}\left(\varepsilon_{e}\right)$ are the experimental stress. The objective of the optimization is to find the best combination of model parameters that minimize the function $f$. The optimization toolbox of matlab is considered here for minimization.

\section{Results and discussion}

The difference-stress method is used in the present work to identify the viscoelastic parameters, and then the elastic parameters are determinate following the result of viscoelastic stress. In the study of Ippili R.K. [8], the viscoelastic parameters are depending on the test conditions. But in the present paper, the dimensionless viscoelastic parameters are considered to predict the foams behaviour under any test conditions [7]. The difference-stress model result of test 1 is shown in Fig.2. The 
dimensionless viscoelastic parameters are given by Table 2 and Table 3. The viscoelastic stress result is shown in Fig. 3.

Table 2. Relationship between Dimensional and dimensionless parameters

\begin{tabular}{|l|l|l|l|}
\hline Dimensional & Dimensionless & Dimensional & Dimensionless \\
\hline$Y(1)=\operatorname{Re}\left(\beta_{1}\right)$ & $X(1)=Y(1) T$ & $Y(5)=\operatorname{Re}\left(\beta_{2}\right)$ & $X(5)=Y(5) T$ \\
\hline$Y(2)=\operatorname{Im}\left(\beta_{1}\right)$ & $X(2)=Y(2) T$ & $Y(6)=\operatorname{Im}\left(\beta_{2}\right)$ & $X(6)=Y(6) T$ \\
\hline$Y(3)=\operatorname{Re}\left(a_{1}\right)$ & $X(3)=\frac{Y(3)}{\sigma_{e x_{-} \max }} \varepsilon_{\max } T$ & $Y(7)=\operatorname{Re}\left(a_{2}\right)$ & $X(7)=\frac{Y(7)}{\sigma_{e x_{-} \max }} \varepsilon_{\max } T$ \\
\hline$Y(4)=\operatorname{Im}\left(a_{1}\right)$ & $X(4)=\frac{Y(4)}{\sigma_{e x_{-} \max }} \varepsilon_{\max } T$ & $Y(8)=\operatorname{Im}\left(a_{2}\right)$ & $X(8)=\frac{Y(8)}{\sigma_{e x_{-} \max }} \varepsilon_{\max } T$ \\
\hline
\end{tabular}

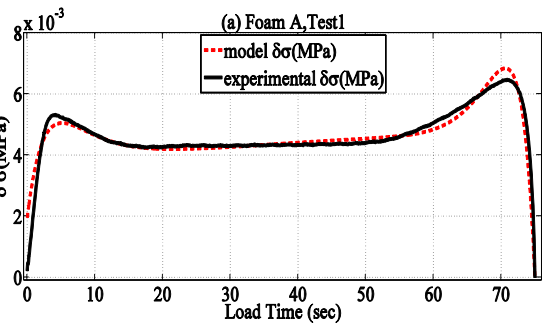

Fig.2 Difference stress model result

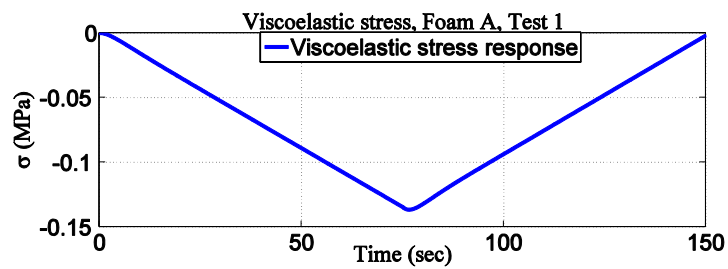

Fig.3 Viscoelastic result

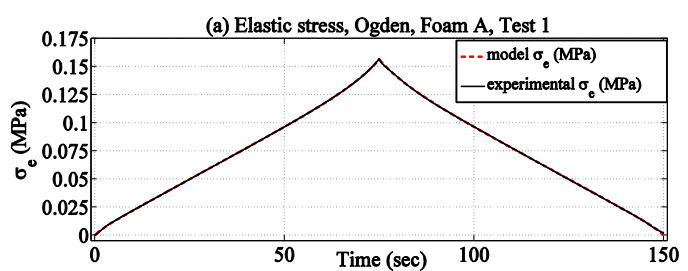

Fig.4 The Ogden model elastic result

Table 3. Dimensionless viscoelastic parameters of foam A in three tests

\begin{tabular}{|c|c|c|c|c|c|c|c|c|}
\hline & $X(1)$ & $X(2)$ & $X(3)$ & $X(4)$ & $X(5)$ & $X(6)$ & $X(7)$ & $X(8)$ \\
\hline Test 1 & 41.72 & 0.174 & -219.81 & 22023.94 & 42.64 & 0.178 & -223.62 & 22027.07 \\
\hline Test 2 & 40.59 & 0.173 & -215.79 & 22023.94 & 41.42 & 0.174 & -217.36 & 22027.07 \\
\hline Test 3 & 43.38 & 0.173 & -214.46 & 22023.94 & 44.38 & 0.178 & -218.35 & 22027.07 \\
\hline
\end{tabular}

As the viscoelastic parameters have already been obtained in table 3, the elastic behaviour described by hyperelastic models mentioned above is now considered. Fig.4 shows the elastic result of Ogden model $(\mathrm{N}=3)$. The difference-method is based on the symmetry condition of elastic stress, so it is necessary to verify the elastic parameters to satisfy this assumption. Fig.4 shows that the model elastic stresses meet the symmetry condition.

Table 4. Ogden model elastic parameters

\begin{tabular}{|l|l|l|l|l|l|l|}
\hline & $\mu_{1}[\mathrm{MPa}]$ & $\alpha_{1}$ & $\mu_{2}[\mathrm{MPa}]$ & $\alpha_{2}$ & $\mu_{3}[\mathrm{MPa}]$ & $\alpha_{3}$ \\
\hline Test 1 & 2.81 & 1.663 & -2.798 & 1.61 & 0.0031 & 38.28 \\
\hline Test 2 & 9.22 & 1.643 & -9.208 & 1.63 & 0.0028 & 38.23 \\
\hline Test 3 & 12.06 & 1.641 & -12.05 & 1.63 & 0.0030 & 38.21 \\
\hline
\end{tabular}

Table 5. Polynomial model elastic parameters

\begin{tabular}{|l|l|l|l|l|l|l|l|}
\hline & C10 [MPa & C01 $[\mathrm{MPa}]$ & C11 $[\mathrm{MPa}]$ & $\mathrm{C} 20[\mathrm{MPa}]$ & $\mathrm{C} 02[\mathrm{MPa}]$ & $\mathrm{D} 1$ & $\mathrm{D} 2$ \\
\hline Test 1 & -2.02 & 1.22 & -0.038 & -0.221 & 0.0056 & 1.182 & 0.251 \\
\hline Test 2 & -1.44 & 0.863 & -0.029 & -0.151 & 0.0042 & 0.879 & 0.205 \\
\hline Test 3 & -1.83 & 1.11 & -0.034 & -0.199 & 0.0051 & 1.069 & 0.232 \\
\hline
\end{tabular}

Table 6. Reduced polynomial model elastic parameters

\begin{tabular}{|l|l|l|l|l|l|l|}
\hline & C10 [MPa] & C20 [MPa] & C30 [MPa & D1 & D2 & D3 \\
\hline Test 1 & -0.068 & 0.0099 & $-6.58 \mathrm{E}-4$ & 0.202 & 0.127 & 0.038 \\
\hline Test 2 & -0.062 & 0.0088 & $-5.69 \mathrm{E}-4$ & 0.187 & 0.114 & 0.038 \\
\hline Test 3 & -0.064 & 0.0093 & $-6.07 \mathrm{E}-4$ & 0.185 & 0.119 & 0.037 \\
\hline
\end{tabular}

The elastic parameters of Ogden model are given in Table 4. Table 5 and Table 6 show the elastic parameters of Polynomial model $(\mathrm{N}=2)$ and Reduced polynomial model $(\mathrm{N}=3)$, respectively.

Fig. 5 shows the global result of Ogden viscoelastic model. It can be seen in Fig.5 that the model curve lie between the maximum and minimum curves in experimental envelops. The model result shows a good agreement with experimental data. 


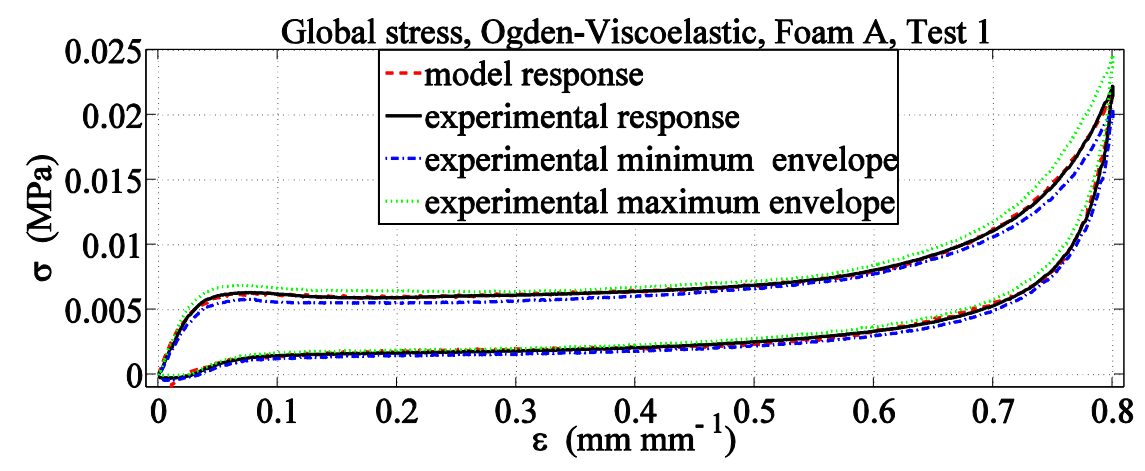

Fig.5 Global result of Ogden-viscoelastic model

The parameters results are a mean of $\mathrm{N}$ test samples (Table 1) which is determined to ensure the statistical quality of all identified parameters. This quality is reviewed through the set at $95 \%$ confidence level and the statistical limit error 'SLE' which shall not exceed $10 \%$.

For the comparison of these three models, the errors between the model result and experimental results are considered (Table 7). Ogden viscoelastic model (with $\mathrm{N}=3$ ) has the least parameters (6 parameters) and has the minimum mean errors in three tests. So, the Ogden viscoelastic model is better than the rest two models.

Table 7. Error results of three visco-hyperelastic models

\begin{tabular}{|l|l|l|l|l|}
\hline \multicolumn{2}{|l|}{ Model } & Ogden-viscoelastic & Polynomial-viscoelastic & Reduced polynomial -viscoelastic \\
\hline \multicolumn{2}{|l|}{ Number of parameters } & 6 & 7 & 6 \\
\hline \multirow{3}{*}{ Foam A } & Test 1 & $2.57 \%$ & $4.25 \%$ & $5.33 \%$ \\
\cline { 2 - 6 } & Test 2 & $2.96 \%$ & $3.64 \%$ & $4.64 \%$ \\
\cline { 2 - 6 } & Test 3 & $3.14 \%$ & $4.34 \%$ & $5.38 \%$ \\
\hline
\end{tabular}

\section{Conclusion}

This paper has presented numerous experiments of polyurethane foam in three different strain rates for loading-unloading uniaxial compression tests. In order to predict the mechanical behaviour of polyurethane foam, three visco-hyperelastic models are studied. Three hyperelastic models are used to describe the elastic behaviour of foams and a hereditary integral model is proposed here to predict the viscoelastic behaviour of foams. A difference-stress method is mentioned to identify the dimensionless viscoelastic parameters. The predictions based on the proposed visco-hyperelastic constitutive model compare well with the experimental data. The errors between the models and experimental data have been analyzed. The results show that the ogden-viscoelastic model with a three-term expression, the polynomial viscoelastic form with $\mathrm{N}=2$ and the reduced polynomial viscoelastic model with $\mathrm{N}=3$ can predict the quasi-static mechanical behaviour of polyurethane foams even under large strain compression. Among the three models, Ogden's viscoelastic model provides the best comparison between the errors and the number of parameters.

\section{References}

[1] Mooney M. Journal of Applied Physics. 11. (1940). 582.

[2] Rivlin RS. Philosophical Transitions of the Royal Society of London. A 240. (1948). 459490.

[3] Rivlin RS. Proceedings of the Cambridge Philosophical Society. 45. (1949). 485.

[4] Ogden RW. Proceedings of the Royal Society of London. 326. (1972). 565-584.

[5] Ogden RW. Proceedings of the Royal Society of London. 328. (1972). 567-583.

[6] Yang LM, Shim VPW. International Journal of Impact Engineering. 30. (2004). 1099-1110.

[7] Jmal H, Dupuis R, Aubry E. Journal of Cellular Plastics. 47. (2011). 447-465.

[8] Ippili RK, Davies P, Balaj AK, Hagenmeyer L. International Journal of Industrial Ergonomics. 38. (2008). 368-383. 